\title{
A Feature Extraction Algorithm of Damaged Military Area Based on Fuzzy Connectedness
}

\author{
Ya-Hong ZHU, Rui WANG, Ji-Ping CAO, Cong-Wei WU \\ Xi'an Research Institution of Hi-Tech, Hong Qing Town, Xi'an City, Shanxi Province, 710025, China \\ zyhbingchen@163.com
}

Keywords: Fuzzy Connectedness, Region Growing, Feature Extraction.

\begin{abstract}
A region growing algorithm based on fuzzy connectedness is proposed in this thesis to solve the military targets' damage problems that the traditional region growing algorithm couldn't handle properly. In this algorithm, the fuzzy connectedness is regarded as the measurement of level of similarity between the growing point and the seed point, which helps to keep the fuzziness among pixels, and it grows according to the fuzzy connectedness in the order - from strong to weak to make the growing results insensitive to the selection of seed points. Besides this, the algorithm combines the gray level fluctuation and USAN area and regards it as the growth rule, which helps to adjust the gray level changes in target regions and the better edge positioning effect is achieved. The experimental results show the efficiency of the algorithm.
\end{abstract}

\section{Introduction}

In remote sensing images, military targets are the artificial structures that could directly or indirectly be used in war and influence the process and the ending of the war, such as the important transportation junctions like airports, highways and bridges, the artificial structures like command buildings, power plants and oil depots. After the military targets are attacked, for the influences of ammunition explosion, the damaged areas would appear which could be shown that the military targets have changed in grayscale, texture, shape and structure in remote sensing images. The gray value of the damaged area information usually corresponds with the minimum value in the local areas because of the low mean gray value. Therefore, the region growing algorithm is often adopted for the feature extraction. It's difficult to extract the damage when adopting the traditional region growing algorithm because of the varieties of damages in the actual military targets, the large area scales, the complex shapes and the vague boundaries. As a consequence, this thesis puts forward the region growing algorithm based on the fuzzy connectedness on account of the poor dispose of the fuzziness between damaged areas and backgrounds as well as the growth results choosing the seed point when the traditional region growing algorithm is used to extract the military targets' damage.

\section{The Region Growing Algorithm Based on Fuzzy Connectedness}

\section{The Similarity Criterion Based on Fuzzy Connectedness}

Fuzziness should be kept when measuring the similarity relation between pixels in images. According to this thought ${ }^{[6-8]}$. Udupa proposes $n$ digital space fuzzy target theory, and according to this theory, the similarity criterion based on fuzzy connectedness could be obtained.

Suppose $R$ denotes the area to grow, $a$ denotes a seed point in its interior and any point in its image as shown in figure 1 . If the area $R$ contains point $b$, the path $P_{a b}$ must exist from the seed point $a$ to the image point $b$. From the conception of the fuzzy connectedness, the value of $\mu_{K}(a, b)$ is unique and it's the best path connectivity value in all the paths. From the symmetry of the fuzzy connectedness, if $b$ is set as the seed point in the area, the path $P_{b a}$ from the seed point $b$ to the image ${ }^{a}$ must also exist at the same time. The value of the path's fuzzy connectedness is $\mu_{K}(b, a)=\mu_{K}(a, b)$, that is, the fuzzy connectedness is regarded as the measure of estimating the 
level of similarity between the growing point and the seed point, and it is set as the measure of controlling its growth. The process of its growth has the feature of symmetry, the point $a$ could grow well if $b$ is set as the seed point. Similarly, the process of the growth also has the features of reflexivity and transitivity.

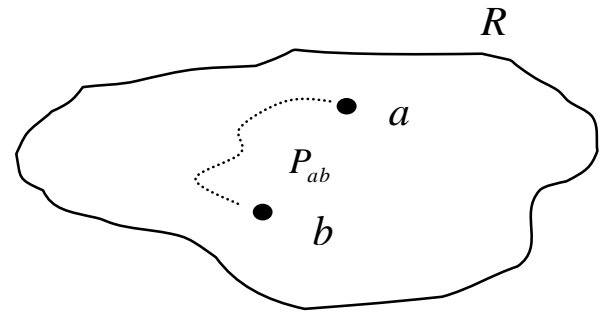

Fig.1 The Schematic Diagram of Growth Based on Fuzzy Connectedness

\section{The Growing Criteria Based on the Combination of Gray Level Fluctuation Control and USAN Area}

In order to extract the damage parts, the region growing criteria should satisfy the following conditions $^{[9,10]}$ :

(1) It has a certain adaptation in the target's gray level distribution,

(2) It has a definite edge positioning abilities and the algorithm stops growing at the edge position.

Therefore, this thesis puts forward a growing criteria based on the combination of the gray level fluctuation control and USAN (Univale Segment Assimilating Nucleus).

\section{The Experimental Result and the Analysis}

The actual damaged image is lack of the true value data, therefore, the thesis chooses the brain images similar to the damages in the remote sensing images in shape and gray level distribution to analyze and compare. The alba area in MR brain section picture has complex shapes, and it has fuzzy boundaries with the ectocinerea and the cerebrospinal fluid, which is similar to the damage extraction in this thesis. Picture 2 shows human brain's MR image, of which map (a) is the original image, map (b) is the alba's corresponding true value image in the original image, the size of the original image and the true value image is $128 \times 128$. In order to verify the effectiveness of the algorithm in this thesis, this article would respectively adopt the algorithm in this thesis and that of the document [1] to extract the alba area in the brain section. In order to compare the sensibility of the two algorithms to the seed point positions, the four positions in the upper left, upper right, bottom left and lower right in the alba area are extracted randomly, as shown in the picture 3(a) (d). These points as the initial seed points of the region growing algorithm grow, and the growing results of these two algorithms are respectively shown in the picture 3(e) (h) and 3(i) (l), whereby the picture 3(e) (h) denotes the result of this article's algorithm, that is, $T_{g}=25$ and $T_{\text {usan }}=0.7$, and the picture 3(i) (1) denotes the result in the document [1].

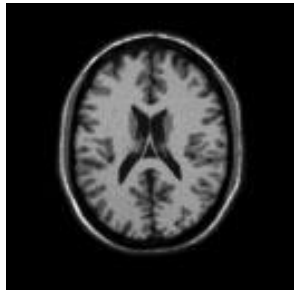

(a) MR Slice Image

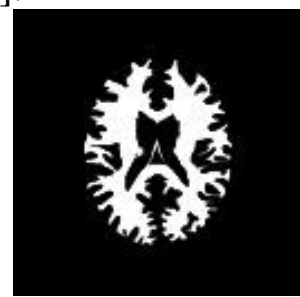

(b) True Value Image

Fig.2 Human Brain MR Slice Image and True Value Image 


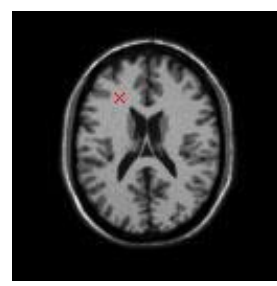

(a)



(e)



(i)

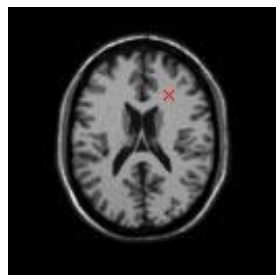

(b)

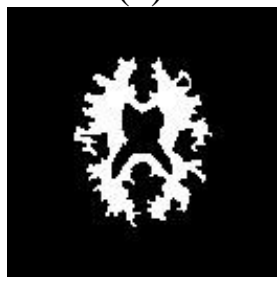

(f)

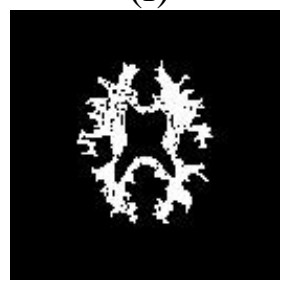

(j)

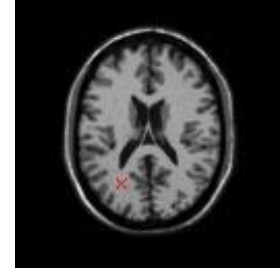

(c)

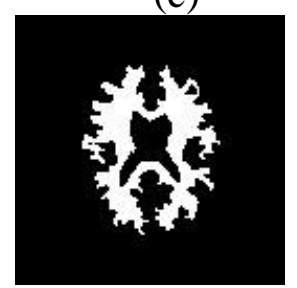

(g)

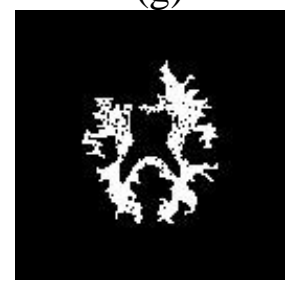

(k)



(d)

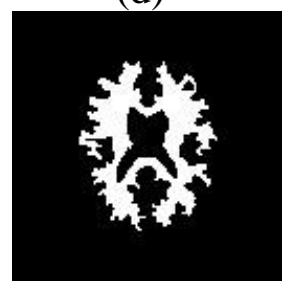

(h)



(1)

Fig.3 The Comparison of Extraction Results of Algorithms in this Article and in Document [1]

As can be seen from the region growing results of the two algorithms in Picture 3, the result extracted from this thesis' algorithm is obviously better than that from the document [1]. For the algorithm of Document [1], under-segmentation appears in the extraction of the alba area with the reasons that the algorithm in Document [1] supposes the gray level of the area-of-interest satisfies Gaussian distribution, but the actual image couldn't satisfy this assumption well. This article adopts the growing criteria of the combination of the gray level fluctuation control and USAN area, and takes into account the gray level fluctuation and the edge positioning ability in the area-of-interest. Therefore, it helps to adapt the object area's gray level change and gets better edge positioning effects.

In order to estimate the two algorithms, FOM (Figure of Merit) is adopted with the following definition:

$$
F O M=\left[\left(1-\frac{\left|C_{r} \otimes C_{G}\right|}{|C|}\right) \times 100\right]
$$

Whereby, $C_{G}$ denotes the truth-value, $C_{r}$ denotes the extracted result, $|C|$ denotes the power of Set $C, \otimes$ denotes the vision of the binary image or the operation. FOM denotes the matching degree of the original target area $C_{G}$ and the target area $C_{r}$.

The Picture 4 shows FOM of the two algorithms, of which the abscissa denotes the selected locations of different initial seed points, the ordinate denotes the matching degree of the original target area $C_{G}$ and the target area $C_{r}$, the higher the resulting value is, the closer the result from the algorithm goes towards the original target area, and the maximum value is 1 . The blue full line and the red imaginary line respectively denote the matching degree curves between the extracted results and the true value images from the algorithms in this thesis and in Document [1]. 




Fig.4 FOM of the Algorithms in This Thesis and That in [20]

From FOM in Picture 4 and in Document [1], it can be seen that the curve of the algorithm in this thesis is far away upside that in Document [1] with flat distribution, which indicates that the extraction result from the algorithm in this thesis has high precision, and it's insensitive to the selected position of the seed point. However, the algorithm in Document [1] has larger fluctuation of curve, which indicates that the extraction result from the algorithm in Document [1] has low precision, and it's sensitive to the selected position of the seed point. Two algorithms have been shown in Tab.1, that is, the time that the region growing has to use in different seed points' positions.

Tab.1 The Comparison of the Time Used of Algorithms in This Thesis and That in [1]

\begin{tabular}{llcc}
\hline $\begin{array}{c}\text { Number of } \\
\text { Position }\end{array}$ & Coordinate & $\begin{array}{c}\text { Algorithm in } \\
\text { Thesis (Second) }\end{array}$ & $\begin{array}{c}\text { Algorithm in Document [20] } \\
\text { (Second) }\end{array}$ \\
\hline 1 & $(52,42)$ & 2.1203 & 3.9010 \\
2 & $(77,39)$ & 2.1201 & 3.8907 \\
3 & $(50,86)$ & 2.1200 & 4.0110 \\
4 & $(81,87)$ & 2.1101 & 4.0061 \\
\hline
\end{tabular}

From Tab.1, it could be seen that the time efficiency in this thesis is superior to that in Document [1] with the time of one half than that of the latter one. The reasons for this situation are that twice region growing are needed in Document [1], the model parameter is estimated in the first time and the region growing proceeds in the second time, but in this thesis, one algorithm is needed and the calculated amount is widely reduced.

\section{Conclusion}

This thesis studies the fact that the region growing algorithm extracts the damage in images. First, according to the characteristics that the military targets' damage possess, for example, the complex shapes, the fuzzy boundaries and the larger inner gray level fluctuation scales, the thesis propose the region growing algorithm based on fuzzy connectedness. In the algorithm that the thesis puts forward, the fuzzy connectedness is regarded as the measurement of level of similarity between the growing point and the seed point, and according to the fuzzy connectedness between the seed point, the growing point is controlled to grow in the order - from strong to weak. This algorithm better keeps the fuzziness among pixels, lowers the sensibility of the growing results to the selections of seed points. Besides this, the algorithm combines the gray level fluctuation and USAN area and regards it as the growth judgment condition, which helps to adjust the gray level changes in target 
regions and the better edge positioning effect is achieved. The experimental results show that the region growing algorithm based on fuzzy connectedness that this thesis puts forward is insensitive to the selection of seed points, and it has better edge positioning abilities with preferable man-machine interaction in the damage extraction.

\section{References}

[1] Li Xuhui, Ci Linlin, Liu Jianhua, et al. Study of Damage Extraction Technology Based on Remote Sensing Image [J]. Automatic Command and Computers, 2008, 3: 51 55.

[2] Zhu Yahong, Wang Mingle, Yang Xiande. The Feature Extraction Algorithm Based on Road Damage Image Information [J]. Projectile and Guidance Journal, 2011, 4:125-127.

[3] Pohle R, Toennies K D. A New Approach for Model-based Adaptive Region Growing in Medical Image Analysis [A]. In: Proceedings of the 9th International Conference on Computer Analysis and Patterns [C]. Warsaw: 2001. 238 246.

[4] Shu-Yen Wan, W. E. Higgins. Symmetric Region Growing [J]. Ieee Transactions On Image Processing, 2003, 12(9): 1007 1015.

[5] Jayaram K. Udupa, Supun Samarasekera. Fuzzy Connectedness and Object Definition: Theory, Algorithms, and Applications in Image Segmentation [J]. Graphical Models and Image Processing, 1996, 58(3): 246 261.

[6] Jayaram K. Udupa. Fuzzy Connectedness and Image Segmentation [J]. Proceedings Of The Ieee, 2003, 91(10): 1649 1669.

[7] Smith SM, Brady JM. SUSAN-A New Approach to Low Level Image Processing [J]. International Journal Of Computer Vision, 1997, 23(1): 45 78.

[8] Roberts L G. Machine Perception of Three-dimension Solids [A]. Optical and Electro-Optimal Information Processing [C]. Cambridge: MIT Press, 1965.

[9] Zhu Chaojie, Dong Guangjun, Wang Renli. Study of Detection Method of Target Changes Based on Feature Extraction [J]. Marine Charting, 2007, 27(2): 50 52.

[10] Cai Hongping, Jiang Yongmei, Su Yi. The Location Method of Oil Depot Clustering Based on Region Growing Theory [J]. Journal of Remote Sensing, 2006, 10(3): 415 419.

[11] Li Guiqin, Yin Dong, Xue Chenrong. Study of Identification Method of Road and Bridge Based on Region Growing [J]. Computer Engineering and Application, 2007, 43(6): 216 218.

[12] Li Xuhui, Ci Linlin, Liu Jianhua, et al. Study of Damage Extraction Technology Based on Remote Sensing Image [J]. Automatic Command and Computers, 2008, 3: 51 55.

[13] Yan D M, Zhao Zh M. Road Detection from Quickbird Fused Image Using IHS Transform and Morphology [A]. IEEE International Geoscience and Remote Sensing Symposium [C]. 2003. $3967 \sim 3969$. 